\title{
Insulin sensitivity, insulin release and glucagon-like peptide-1 levels in persons with impaired fasting glucose and/or impaired glucose tolerance in the EUGENE2 study
}

\author{
M. Laakso • J. Zilinskaite $\cdot$ T. Hansen • \\ T. Welløv Boesgaard $\cdot$ M. Vänttinen • A. Stančáková • \\ P.-A. Jansson • F. Pellmé • J. J. Holst • T. Kuulasmaa • \\ M. L. Hribal • G. Sesti • N. Stefan • A. Fritsche • \\ H. Häring • O. Pedersen • U. Smith • \\ for the EUGENE2 Consortium
}

Received: 27 June 2007 / Accepted: 5 November 2007 / Published online: 14 December 2007

(C) Springer-Verlag 2007

\begin{abstract}
Aims/hypothesis We examined the phenotype of individuals with impaired fasting glucose (IFG) and/or impaired glucose tolerance (IGT) with regard to insulin release and insulin resistance.

Methods Non-diabetic offspring ( $n=874$; mean age $40 \pm$ 10.4 years; BMI $26.6 \pm 4.9 \mathrm{~kg} / \mathrm{m}^{2}$ ) of type 2 diabetic patients from five different European Centres (Denmark, Finland, Germany, Italy and Sweden) were examined with regard to insulin sensitivity (euglycaemic clamps), insulin release (IVGTT) and glucose tolerance (OGTT). The levels of glucagon-like peptide-1 (GLP-1) and gastric inhibitory polypeptide (GIP) were measured during the OGTT in 278 individuals.

Results Normal glucose tolerance was found in 634 participants, while 110 had isolated IFG, 86 had isolated IGT and
\end{abstract}

M. Laakso · J. Zilinskaite · M. Vänttinen · A. Stančáková •

T. Kuulasmaa

Department of Medicine, University of Kuopio,

Kuopio, Finland

T. Hansen $\cdot$ T. W. Boesgaard $\cdot$ O. Pedersen

Steno Diabetes Center,

Gentofte, Denmark

P.-A. Jansson $\cdot$ F. Pellmé $\cdot$ U. Smith

The Lundberg Laboratory for Diabetes Research,

Department of Molecular and Clinical Medicine,

The Sahlgrenska Academy at Gothenburg University,

Gothenburg, Sweden

J. J. Holst

Department of Biomedical Sciences, University of Copenhagen,

Copenhagen, Denmark
44 had both IFG and IGT, i.e. about $28 \%$ had a form of reduced glucose tolerance. Participants with isolated IFG had lower glucose-corrected first-phase $(0-10 \mathrm{~min})$ and higher second-phase insulin release (10-60 min) during the IVGTT, while insulin sensitivity was reduced in all groups with abnormal glucose tolerance. Similarly, GLP-1 but not GIP levels were reduced in individuals with abnormal glucose tolerance.

Conclusions/interpretation The primary mechanism leading to hyperglycaemia in participants with isolated IFG is likely to be impaired basal and first-phase insulin secretion, whereas in isolated IGT the primary mechanism leading to postglucose load hyperglycaemia is insulin resistance. Reduced GLP-1 levels were seen in all groups with abnormal glucose tolerance and were unrelated to the insulin release pattern during an IVGTT.

M. L. Hribal · G. Sesti

Department of Experimental and Clinical Medicine,

University Magna Græcia of Catanzaro,

Catanzaro, Italy

N. Stefan $\cdot$ A. Fritsche $\cdot$ H. Häring

Department of Internal Medicine,

Division of Endocrinology,

Diabetology, Nephrology, Vascular Medicine

and Clinical Chemistry, University of Tubingen,

Tubingen, Germany

U. Smith $(\bowtie)$

The Lundberg Laboratory for Diabetes Research,

Department of Molecular and Clinical Medicine,

Sahlgrenska University Hospital,

41345 Gothenburg, Sweden

e-mail: ulf.smith@medic.gu.se 
Keywords Gastric inhibitory polypeptide .

Glucagon-like peptide-1 · Glucose · Insulin · Insulin release Insulin sensitivity · Offspring · Type 2 diabetes

\begin{tabular}{|c|c|}
\hline \multicolumn{2}{|c|}{ Abbreviations } \\
\hline EUGENE2 & $\begin{array}{l}\text { European Network on Functional Genomics } \\
\text { of Type } 2 \text { Diabetes }\end{array}$ \\
\hline GIP & gastric inhibitory polypeptide \\
\hline GLP-1 & glucagon-like peptide-1 \\
\hline HOMA-B & $\begin{array}{l}\text { homeostasis model assessment of beta cell } \\
\text { function }\end{array}$ \\
\hline HOMA-IR & $\begin{array}{l}\text { homeostasis model assessment of insulin } \\
\text { resistance }\end{array}$ \\
\hline IFG & impaired fasting glucose \\
\hline IGT & impaired glucose tolerance \\
\hline NGT & normal glucose tolerance \\
\hline
\end{tabular}

\section{Introduction}

Type 2 diabetes is a common disease affecting about 3 to $5 \%$ of those in western populations. Major pathophysiological mechanisms for type 2 diabetes are insulin resistance and impaired insulin secretion; both of these abnormalities also predict the development of type 2 diabetes in prospective studies $[1,2]$.

The insulin response to a hyperglycaemic stimulation is biphasic [3]. Insulin concentrations in the plasma increase rapidly to a peak at 2 to $4 \mathrm{~min}$, decrease to a nadir at 10 to $15 \mathrm{~min}$ and then gradually increase over 120 to $180 \mathrm{~min}$. The initial response is referred to as first-phase insulin release and the subsequent increase in insulin secretion represents the second-phase insulin release [4]. Normal islets compensate for insulin resistance $[5,6]$, making it essential, in any evaluation of the appropriateness of insulin secretion, to take prevailing insulin sensitivity into account.

The new entities of impaired fasting glucose (IFG) and impaired glucose tolerance (IGT) have triggered studies on the mechanisms for increased fasting and postprandial (or postglucose load) glucose. IFG was first introduced by the American Diabetes Association [7] and defined as a fasting plasma glucose level between 6.1 and $7.0 \mathrm{mmol} / 1$. Subsequently, the lower cut-off point of plasma glucose was changed to $5.6 \mathrm{mmol} / \mathrm{l}$ [8]. IGT is defined on the basis of an OGTT as a $2 \mathrm{~h}$ plasma glucose level between 7.8 and $11.1 \mathrm{mmol} / \mathrm{l}$ and normal glucose tolerance (NGT) as fasting plasma glucose $<5.6 \mathrm{mmol} / 1$ and $2 \mathrm{~h}$ plasma glucose $<7.8 \mathrm{mmol} / 1$ [9].

Previous studies have given conflicting data on the association of IFG and IGT with insulin resistance and impaired insulin secretion [10-20]. In a study of Pima Indians, participants with isolated IFG and isolated IGT showed similar impairments in insulin sensitivity, but those with isolated IFG had a more pronounced defect in early insulin release and higher endogenous glucose output [10]. Tripathy et al. [11], on the other hand, concluded that IFG was characterised by insulin resistance, whereas persons with IGT had impaired insulin secretion. Similar findings were reported by Hanefeld et al. [15]. Van Haeften et al. [14] concluded that the early stages of glucose intolerance (IFG) are associated with disturbances in beta cell function, whereas insulin resistance is more marked at later stages (participants with both IFG and IGT). Another study by the same research group [19] reported that IFG is due to impaired basal insulin secretion and preferential resistance to the suppressive effect of insulin on hepatic glucose production, whereas IGT mainly results from reduced second-phase insulin release accompanied by peripheral insulin resistance. In a recent study, isolated IFG was associated with normal insulin secretion but impaired insulin sensitivity, whereas in persons with isolated IGT both insulin secretion and insulin sensitivity were impaired [20]. These conflicting results are probably due to different study designs, different methods of measuring insulin resistance and insulin secretion, small sample size, heterogeneity of IFG and IGT, and/or failure to classify patients on the basis of isolated IFG and isolated IGT.

The European Network on Functional Genomics of Type 2 Diabetes (EUGENE2) consortium has collected data on 874 carefully phenotyped non-diabetic offspring of type 2 diabetic patients from five different centres in Europe. This large database gives us an opportunity to investigate the role of insulin resistance and impaired insulin secretion as primary abnormalities in the pathophysiology of IFG and IGT in persons at high risk of type 2 diabetes.

\section{Methods}

Participants The participants included in this study were healthy, non-diabetic offspring of patients with type 2 diabetes. One of the parents had to have type 2 diabetes and the other parent NGT in an OGTT and/or no history of type 2 diabetes in the family. The probands were randomly selected among type 2 diabetic patients living in the regions of five centres in Europe. They were recruited over a 4 year period through advertisements in public media and in the hospitals. The acceptance rate of volunteers was at least $70 \%$ in the different centres. Altogether, 874 offspring were included in the study from the following centres: Catanzaro, Italy $(n=130)$, Copenhagen, Denmark $(n=278)$, Gothenburg, Sweden $(n=100)$, Kuopio, Finland 
( $n=217)$ and Tubingen, Germany $(n=149)$. The number of families with one to four offspring or more than four offspring was 109/0 in Catanzaro, 30/26 in Copenhagen, 97/0 in Gothenburg, 132/0 in Kuopio and 142/0 in Tubingen. The study protocol was approved by the appropriate Institutional Review Boards. All study participants gave informed consent.

Measurements and metabolic studies All centres followed the same protocol. Blood pressure was measured with a mercury sphygmomanometer in the sitting position after a $5 \mathrm{~min}$ rest. Height and weight were measured to the nearest $0.5 \mathrm{~cm}$ and $0.1 \mathrm{~kg}$, respectively and BMI $\left(\mathrm{kg} / \mathrm{m}^{2}\right)$ calculated. Waist and hip circumferences were measured to the nearest $0.5 \mathrm{~cm}$. Fasting (overnight) blood samples were drawn followed by an OGTT (75 $\mathrm{g}$ glucose) to evaluate glucose tolerance status (blood samples taken at 0 , 30, 90 and $120 \mathrm{~min}$ ). On a second occasion, an IVGTT was performed to determine the first-phase insulin secretion capacity after an overnight fast. A bolus of glucose $(300 \mathrm{mg} / \mathrm{kg}$ in a $50 \%$ [wt/vol.] solution) was given within $30 \mathrm{~s}$ into the antecubital vein. Arterialised venous blood samples for the measurement of plasma glucose and insulin were drawn at $-5,0,2,4,6,8,10,20,30,40,50$ and $60 \mathrm{~min}$. At $60 \mathrm{~min}$ after the glucose bolus, a hyperinsulinaemic-euglycaemic clamp was started (insulin infusion $240 \mathrm{pmol} \mathrm{m}{ }^{-2} \min ^{-1}$ ) and carried out for the next $120 \mathrm{~min}$ to evaluate insulin sensitivity [21]. This design has been previously validated [22].

Blood glucose was clamped at $5.0 \mathrm{mmol} / \mathrm{l}$ for the next 120 min by infusion of $20 \%$ glucose at various rates according to the blood glucose measurements performed at 5 min intervals. The mean amount of glucose infused during the last hour was used to calculate the rate of whole-body glucose uptake. In the Copenhagen centre, the euglycaemic clamp was not performed; instead, insulin sensitivity was measured by a tolbutamide-modified frequently sampled IVGTT to measure insulin sensitivity (data not shown).

We analysed the results in four categories of glucose tolerance: NGT ( $n=634$; fasting plasma glucose $<5.6 \mathrm{mmol} / \mathrm{l}$ and $2 \mathrm{~h}$ plasma glucose $<7.8 \mathrm{mmol} / \mathrm{l})$; isolated IFG $(n=110$; fasting plasma glucose $5.6-6.9 \mathrm{mmol} / \mathrm{l}$ and $2 \mathrm{~h}$ plasma glucose $<7.8 \mathrm{mmol} / \mathrm{l})$; isolated IGT $(n=86$; fasting glucose $<5.6 \mathrm{mmol} / \mathrm{l}$ and $2 \mathrm{~h}$ plasma glucose $7.8-11.0 \mathrm{mmol} / \mathrm{l}$ ); and simultaneous presence of IFG and IGT $(n=44)$.

Laboratory determinations Glucose was measured by the same glucose oxidase method in the different centres. Plasma insulin was measured with different methods and, therefore, the assay applied in Tubingen was used as a reference method. All insulin samples from the Gothenburg centre were measured in Tubingen. The insulin measure- ments in Catanzaro, Copenhagen and Kuopio were standardised by having about 100 fasting and postglucose challenge plasma samples measured in Tubingen. The plasma insulin levels from these three centres were converted to plasma insulin levels corresponding to the Tubingen assay by linear regression analysis which gave the best fit. Cholesterol and triacylglycerol levels from whole serum and from lipoprotein fractions were assayed by automated enzymatic methods (Roche Diagnostics, Mannheim, Germany).

Gastric inhibitory polypeptide (GIP) and glucagon-like peptide-1 (GLP-1) concentrations in plasma were measured during the OGTT in the 278 individuals from Copenhagen after extraction of plasma with $70 \%$ ethanol (vol./vol., final concentration). For the GIP radioimmunoassay [23], we used the C-terminally directed antiserum R 65 , which crossreacts fully with human GIP but not with the so-called GIP 8000 , whose chemical nature and relationship to GIP secretion is uncertain. It reacts fully with the primary metabolite, GIP 3-42. Human GIP and $\left[{ }^{125} \mathrm{I}\right]$-human GIP $(70 \mathrm{MBq} / \mathrm{nmol})$ were used for standards and tracer. The plasma concentrations of GLP-1 were measured [24] against standards of synthetic GLP-1 7-36 amide using antiserum (code no. 89390), which is specific for the amidated C-terminus of GLP-1 and therefore does not react with GLP-1-containing peptides from the pancreas. The results of the assay accurately reflect the rate of secretion of GLP-1 because the assay measures the sum of intact GLP-1 and the primary metabolite, GLP-1 9-36 amide, into which GLP-1 is rapidly converted [25]. For both assays, sensitivity was below $1 \mathrm{pmol} / \mathrm{l}$, intra-assay coefficient of variation below $6 \%$ at $20 \mathrm{pmol} / 1$ and recovery of standard, added to plasma before extraction, about $100 \%$ when corrected for losses inherent in the plasma extraction procedure.

Statistical analysis Data analyses were carried out with SPSS 11.0 for Windows (Chicago, IL, USA). The results for continuous variables are given as means \pm SD. Variables with skewed distribution (insulin, triacylglycerol) were logarithmically transformed for statistical analyses. The areas under the insulin and glucose curves in the OGTT and IVGTT (first-phase insulin release from 0 to $10 \mathrm{~min}$, second-phase insulin release from 10 to $60 \mathrm{~min}$ ) were calculated by the trapezoidal method. The differences between the groups were assessed by ANOVA for continuous variables and by the $\chi^{2}$ test for non-continuous variables. Linear mixed model analysis was applied to adjust for confounding factors. For mixed model analysis, we included the family (sibship) and centre as random factors, the sex as a fixed factor, and both age and BMI as covariates. The Bonferroni correction was used to adjust for multiple comparisons. 


\section{Results}

Baseline characteristics Characteristics, by centre, of the 874 participants are presented in Table 1 . Breakdown by sex was $43 \%$ men and $57 \%$ women ( $p<0.001$ among the centres), age $40.1 \pm 10.4$ years $(p<0.001)$, BMI $26.6 \pm 4.9 \mathrm{~kg} / \mathrm{m}^{2}(p<$ $0.001)$, fasting plasma glucose $5.1 \pm 0.5 \mathrm{mmol} / 1 \quad(p<0.001)$ and $2 \mathrm{~h}$ plasma glucose $6.2 \pm 1.5 \mathrm{mmol} / \mathrm{l}(p=0.082)$. Table 2 shows clinical and laboratory parameters according to different glucose tolerance categories. Altogether, 634 participants had NGT, 110 had isolated IFG, 86 isolated IGT and 44 participants had both IFG and IGT; thus $28 \%$ had a form of reduced glucose tolerance. Participants with abnormal glucose tolerance were significantly older, had higher BMI and larger waist circumference (isolated IFG group) than those with NGT. They also had higher systolic and diastolic blood pressure and total triacylglycerol levels (isolated IGT, IFG/IGT) than participants with NGT. LDLcholesterol was higher and HDL-cholesterol lower in participants with isolated IFG than in normoglycaemic participants, but no difference with respect to total and LDL-cholesterol was found between the other groups.

OGTT data Glucose and insulin responses during the OGTT are shown in Fig. 1. Fasting insulin levels were higher in participants with isolated IFG and in those with IFG/IGT than in participants with NGT. No difference between the groups was observed at $30 \mathrm{~min}$ insulin levels, but at all subsequent time points insulin levels were higher among participants with abnormal glucose tolerance than in those with NGT. The ratio of the glucose:insulin AUC during the OGTT did not differ between the groups.

IVGTT data Figure 2 shows first-phase $(0-10 \mathrm{~min})$ and second-phase (10-60 min) glucose and insulin responses.
Participants with abnormal glucose tolerance had higher insulin levels except at 10 and $20 \mathrm{~min}$.

Figure 3 shows AUCs for first-phase and second-phase glucose and insulin responses. As expected, the first-phase glucose AUC was significantly higher in participants with isolated IFG $(7.5 \%$ [95\% CI $5.2-9.8 \%], p=0.004)$, isolated IGT $(5.8 \%$ [95\% CI $2.8-8.8 \%], p=0.046)$ and with IFG/IGT (13.7\% [95\% CI 9.2-18.2\%], $p=0.038)$ compared with NGT participants. First-phase insulin response was not significantly different between participants with NGT when compared with the other groups. However, when corrected for the ambient glucose concentration, participants with isolated IFG had lower first-phase insulin AUC:glucose AUC ratio than participants with NGT even after adjustment for confounding factors, including insulin sensitivity ( $22.5 \%$ [ $95 \%$ CI $3.9-40.7 \%$ ], $p=0.003$ ). Insulin AUC:glucose AUC ratio was also lower (49.6\% [95\% CI 18.6-80.6\%], $p<0.001)$ among participants with IFG/IGT than in those with NGT. In contrast, the first-phase insulin AUC:glucose AUC ratio did not differ between participants with isolated IGT and NGT. Secondphase insulin AUC was higher among participants with IFG/IGT than in those with NGT $(20.9 \%$ [95\% CI $8.2-$ $33.6 \%], p=0.004)$.

Insulin sensitivity Insulin sensitivity was analysed both with the homeostasis model assessment of insulin resistance (HOMA-IR) [26] and the euglycaemic clamp technique. HOMA-IR was higher in participants with isolated IFG $(17.9 \pm 26.6, p<0.001)$, isolated IGT $(13.3 \pm 1.3, p=0.069)$ and in participants with IFG/IGT $(28.5 \pm 40.8, p<0.001)$ than in those with NGT $(9.5 \pm 6.4)$. HOMA assessment of beta cell function (HOMA-B) [26], was lower in participants with IFG $(27.4 \pm 30.6, p=0.002)$ than in participants with NGT $(34.0 \pm 29.7)$, but HOMA-B did not differ in

Table 1 Characteristics of the participants according to study centre

\begin{tabular}{|c|c|c|c|c|c|c|c|}
\hline & $\begin{array}{l}\text { Gothenburg } \\
(n=100)\end{array}$ & $\begin{array}{l}\text { Catanzaro } \\
(n=130)\end{array}$ & $\begin{array}{l}\text { Copenhagen } \\
(n=278)\end{array}$ & $\begin{array}{l}\text { Kuopio } \\
(n=217)\end{array}$ & $\begin{array}{l}\text { Tubingen } \\
(n=149)\end{array}$ & $p$ value & $\begin{array}{l}\text { Total } \\
(n=874)\end{array}$ \\
\hline Families $(n)$ & 97 & 109 & 56 & 132 & 142 & & \\
\hline Male/female $(n)$ & $24 / 76$ & $45 / 85$ & $121 / 157$ & $104 / 113$ & $83 / 66$ & $<0.001$ & $377 / 497$ \\
\hline NGT $(n)$ & 79 & 104 & 196 & 152 & 103 & 0.097 & 634 \\
\hline Isolated IFG $(n)$ & 1 & 11 & 48 & 34 & 16 & $<0.001$ & 110 \\
\hline Isolated IGT (n) & 19 & 8 & 17 & 22 & 20 & 0.001 & 86 \\
\hline IFG and IGT $(n)$ & 1 & 7 & 17 & 9 & 10 & 0.253 & 44 \\
\hline Age (years) & $40.0 \pm 7.2$ & $38.2 \pm 11.1$ & $42.5 \pm 11.8$ & $35.2 \pm 6.2$ & $44.5 \pm 10.5$ & $<0.001$ & $40.1 \pm 10.4$ \\
\hline Weight (kg) & $74.2 \pm 12.7$ & $78.2 \pm 18.1$ & $77.6 \pm 14.5$ & $77.8 \pm 15.1$ & $82.8 \pm 17.7$ & 0.001 & $78.2 \pm 15.8$ \\
\hline BMI $\left(\mathrm{kg} / \mathrm{m}^{2}\right)$ & $24.8 \pm 3.5$ & $28.0 \pm 5.8$ & $26.3 \pm 4.6$ & $26.2 \pm 4.7$ & $27.9 \pm 5.2$ & $<0.001$ & $26.6 \pm 4.9$ \\
\hline FPG (mmol/l) & $4.8 \pm 0.4$ & $4.9 \pm 0.7$ & $5.2 \pm 0.5$ & $5.2 \pm 0.4$ & $5.1 \pm 0.5$ & $<0.001$ & $5.1 \pm 0.5$ \\
\hline 2-h PG (mmol/l) & $6.3 \pm 1.5$ & $6.0 \pm 1.6$ & $6.1 \pm 1.6$ & $6.3 \pm 1.4$ & $6.5 \pm 1.6$ & 0.082 & $6.2 \pm 1.5$ \\
\hline
\end{tabular}

Unless otherwise indicated, values are means $\pm \mathrm{SD}$

FPG, fasting plasma glucose; 2-h PG, 2 h plasma glucose 
Table 2 Characteristics of the participants according to glucose tolerance status

\begin{tabular}{|c|c|c|c|c|c|c|}
\hline & $\begin{array}{l}\text { NGT } \\
(n=634)\end{array}$ & $\begin{array}{l}\text { Isolated IFG } \\
(n=110)\end{array}$ & $\begin{array}{l}\text { Isolated IGT } \\
(n=86)\end{array}$ & $\begin{array}{l}\text { IFG and IGT } \\
(n=44)\end{array}$ & $\begin{array}{l}\text { Overall } \\
p \text { value }\end{array}$ & $\begin{array}{l}\text { Total } \\
(n=874)\end{array}$ \\
\hline Age (years) & $38.9 \pm 9.9$ & $41.4 \pm 9.7^{\mathrm{a}}$ & $41.8 \pm 10.3^{\mathrm{a}}$ & $50.6 \pm 12.3^{\mathrm{c}}$ & $<0.001$ & $40.1 \pm 10.4$ \\
\hline Sex (men/women) & $253 / 381$ & $69 / 41^{\mathrm{c}}$ & $32 / 54$ & $23 / 21$ & $<0.001$ & $377 / 497$ \\
\hline Weight (kg) & $76.0 \pm 14.7$ & $85.7 \pm 16.8^{\mathrm{c}}$ & $79.3 \pm 16.0$ & $89.4 \pm 15.8^{\mathrm{c}}$ & $<0.001$ & $78.2 \pm 15.8$ \\
\hline BMI $\left(\mathrm{kg} / \mathrm{m}^{2}\right)$ & $26.0 \pm 4.7$ & $27.6 \pm 4.7^{\mathrm{b}}$ & $27.8 \pm 5.3^{\mathrm{b}}$ & $30.7 \pm 5.3^{\mathrm{c}}$ & $<0.001$ & $26.6 \pm 4.9$ \\
\hline Waist circumference $(\mathrm{cm})$ & $86.8 \pm 12.5$ & $94.8 \pm 13.4^{\mathrm{c}}$ & $91.0 \pm 13.2$ & $100.7 \pm 14.1$ & $<0.001$ & $89.0 \pm 13.3$ \\
\hline $\mathrm{SBP}(\mathrm{mmHg})$ & $119.9 \pm 13.0$ & $126.2 \pm 16.0^{\mathrm{b}}$ & $124.7 \pm 15.5^{\mathrm{a}}$ & $139.5 \pm 21.8^{\mathrm{c}}$ & $<0.001$ & $122.2 \pm 14.9$ \\
\hline DBP (mmHg) & $76.5 \pm 10.0$ & $80.3 \pm 11.2^{\mathrm{a}}$ & $79.0 \pm 11.7$ & $85.5 \pm 14.6^{\mathrm{c}}$ & $<0.001$ & $77.7 \pm 10.9$ \\
\hline Smoking (\%) & 31 & $45^{\mathrm{b}}$ & 24 & 32 & 0.015 & 32 \\
\hline Total cholesterol $(\mathrm{mmol} / \mathrm{l})$ & $4.96 \pm 1.00$ & $5.13 \pm 0.95$ & $5.12 \pm 0.92$ & $5.46 \pm 0.95$ & 0.978 & $5.02 \pm 0.99$ \\
\hline LDL-cholesterol (mmol/l) & $3.00 \pm 0.80$ & $3.37 \pm 0.82^{\mathrm{a}}$ & $3.15 \pm 0.80$ & $3.25 \pm 0.56$ & 0.155 & $3.07 \pm 0.80$ \\
\hline HDL-cholesterol (mmol/l) & $1.39 \pm 0.36$ & $1.19 \pm 0.30^{\mathrm{b}}$ & $1.33 \pm 0.29$ & $1.23 \pm 0.34$ & $<0.01$ & $1.35 \pm 0.35$ \\
\hline Total triacylglycerol $(\mathrm{mmol} / \mathrm{l})$ & $1.15 \pm 0.69$ & $1.33 \pm 0.61$ & $1.54 \pm 1.21^{\mathrm{b}}$ & $1.87 \pm 1.53^{\mathrm{b}}$ & 0.001 & $1.25 \pm 0.83$ \\
\hline FPG $(\mathrm{mmol} / \mathrm{l})$ & $4.9 \pm 0.4$ & $5.9 \pm 0.3^{\mathrm{c}}$ & $5.0 \pm 0.4$ & $6.1 \pm 0.4^{\mathrm{c}}$ & $<0.001$ & $5.1 \pm 0.5$ \\
\hline FPI (pmol/l) & $43 \pm 28$ & $67 \pm 91^{\mathrm{b}}$ & $59 \pm 49^{\mathrm{a}}$ & $105 \pm 146^{\mathrm{c}}$ & 0.001 & $51 \pm 56$ \\
\hline FPI (geometric mean) & $3.75 \pm 0.66$ & $3.93 \pm 0.66$ & $3.85 \pm 0.68$ & $4.32 \pm 0.72$ & 0.001 & $3.68 \pm 0.70$ \\
\hline
\end{tabular}

Unless otherwise indicated, values are means $\pm \mathrm{SD}$

The $p$ values for age, sex, weight, BMI and smoking are unadjusted. All other $p$ values are adjusted for age, sex, BMI, centre and familial relationship (sibship)

For categorical variables the $\chi^{2}$ test was applied

${ }^{\mathrm{a}} p<0.05,{ }^{\mathrm{b}} p<0.01,{ }^{\mathrm{c}} p<0.001$ compared with NGT

DBP, diastolic blood pressure; FPI, fasting plasma insulin; FPG, fasting plasma glucose; SBP, systolic blood pressure

participants with isolated IGT (41.7 \pm 34.0$)$ or with IFG/IGT $(40.8 \pm 53.1)$ from that of participants with NGT.

Figure 4 shows insulin sensitivity, evaluated by the euglycaemic clamp, as rates of whole-body glucose uptake according to glucose tolerance status. Participants with isolated IFG $(17.5 \%$ [95\% CI 7.9-27.1\%], $p<0.001)$, isolated IGT $(26.2 \%$ [95\% CI 18.9-33.6\%], $p=0.016)$ and IFG/IGT $(33.2 \%$ [95\% CI $18.8-47.6 \%], p<0.001)$ had significantly lower insulin sensitivity than participants with NGT. Furthermore, participants with isolated IGT had lower insulin sensitivity than those with isolated IFG ( $p=$ 0.041). Participants who had both IFG and IGT had the lowest rates of whole-body glucose uptake. Disposition index was lower among participants with isolated IFG (20.6\% [95\% CI $0-48.1 \%$ ] $p=0.047)$ and isolated IGT $(31.9 \%$ [95\% CI 4.5-59.2\%], $p=0.009)$ than in those with NGT, whereas participants with IFG and IGT had a higher disposition index $(p=0.048)$ than in those with NGT.

Incretin levels Figure 5a shows that GLP-1 levels were lower in participants with any type of abnormal glucose tolerance status than in those with NGT at 15,90 and $120 \mathrm{~min}$; the 0 to $240 \mathrm{~min}$ AUC was also lower $(p=0.024)$. However, there was no difference in GIP levels (Fig. 5b) including 0 to 240 min AUC (data not shown).

Finally, we also analysed all data on the basis of a cut-off point of $6.1 \mathrm{mmol} / \mathrm{l}$ for fasting glucose levels in participants with isolated IGT. The results were not substantially different from those presented above.

\section{Discussion}

People with prediabetes (IFG, IGT) are at increased risk of developing type 2 diabetes $[1,2,27]$ and cardiovascular disease [28]. The definitions of IFG and IGT, based on fasting and $2 \mathrm{~h}$ glucose levels, identify glucose tolerance categories that might have different aetiologies, metabolic profile and prognostic importance. Therefore, the pathogenesis of IFG and IGT has attracted considerable interest, and several studies have tried to define the characteristics of these groups as discussed.

Our study is based on a large cohort of 874 offspring of type 2 diabetic patients, i.e. individuals who are at high risk of developing type 2 diabetes as also supported by the finding that $28 \%$ of this fairly young and non-obese cohort had a form of reduced glucose tolerance. We showed that isolated IFG is characterised by a reduced glucose-adjusted first-phase insulin release (insulin AUC:glucose AUC ratio) as evaluated by an IVGTT $(-22 \%)$, lower basal insulin secretion evaluated by HOMA-B (-19\%) and decreased insulin sensitivity $(-17 \%)$ evaluated by the euglycaemichyperinsulinaemic clamp. In contrast, participants with isolated IGT did not have impaired insulin secretion, but 
a

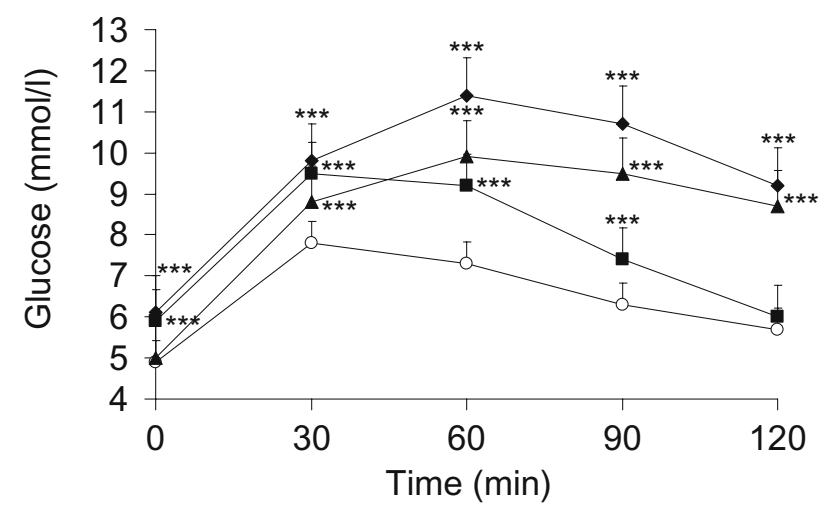

b

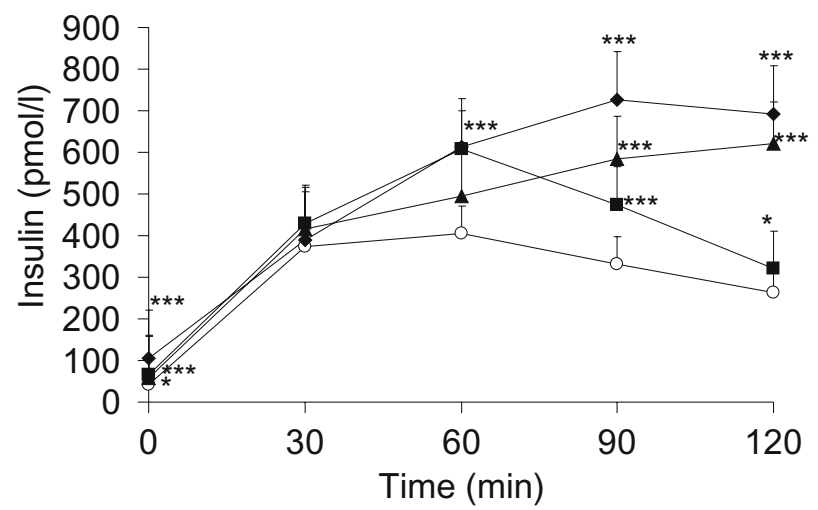

Fig. 1 Glucose (a) and insulin (b) levels in participants with different glucose tolerance during the OGTT. Circles, normal glucose tolerance; squares, isolated IFG; triangles, isolated IGT; diamonds, combined IFG and IGT. $p$ values are adjusted for age, sex, BMI, centre and familial relationship. ${ }^{*} p<0.05, * * * p<0.001$ vs NGT

were more insulin-resistant than participants with isolated IFG. The most severe insulin resistance was found in participants with both IFG/IGT. Thus, our study provides further support for the concept that IFG and IGT are distinct metabolic abnormalities and/or represent the temporal phenotypic development along the path to type 2 diabetes.

Insulin sensitivity was evaluated with HOMA-IR and the euglycaemic clamp. In general, both measurements gave the same information, i.e. that all groups with IGT were more insulin-resistant. However, comparisons between the groups showed some inconsistencies. HOMA-IR was higher in the isolated IFG than in the isolated IGT group, while people with isolated IGT were more insulin-resistant when measured with the euglycaemic clamp. A similar discrepancy has previously been found [16] and supports the conclusion that the HOMA model is a less reliable assessment of insulin sensitivity when comparing groups of patients with isolated IFG and isolated IGT, where, by definition, fasting glucose levels are different.

Interestingly, we found that the release of GLP-1 but not GIP was reduced in all three groups with abnormal glucose tolerance. Reduced incretin effect and reduced GLP-1 levels following a meal have previously been reported in type 2 diabetic participants as well as in those with IGT $[29,30]$, although the significance of this for the dysmetabolic state is unclear. Since the GLP-1 levels were reduced in IFG as well as in IGT participants and these states have a different early insulin response to glucose, it may be concluded that this abnormality is unlikely to account for the glucose intolerance. However, we cannot rule out the possibility that effects of GLP-1 outside the beta cell, i.e. inhibition of glucagon secretion and/or effects on gastric emptying, play a role. In addition, this possibility may provide a mechanism for the future development of the insulin-deficient state that characterises type 2 diabetes. Since not all IFG/IGT participants had reduced GLP-1 levels, individual susceptibility in maintaining beta cell function and in developing frank type 2 diabetes may be related to the ability to release incretins. However, this obviously needs to be addressed in future prospective studies.

The first study investigating the metabolic profile of participants with isolated IFG and isolated IGT did so by using accurate methods for measurement of insulin secre-

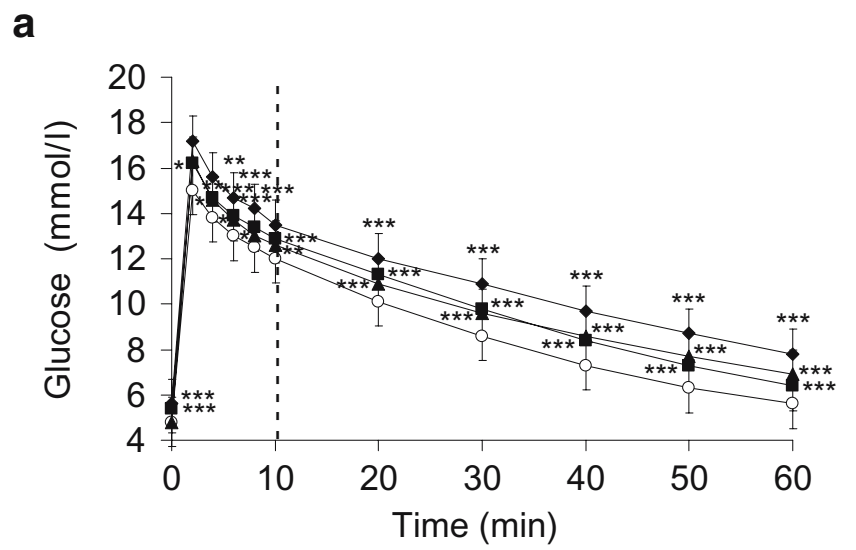

b

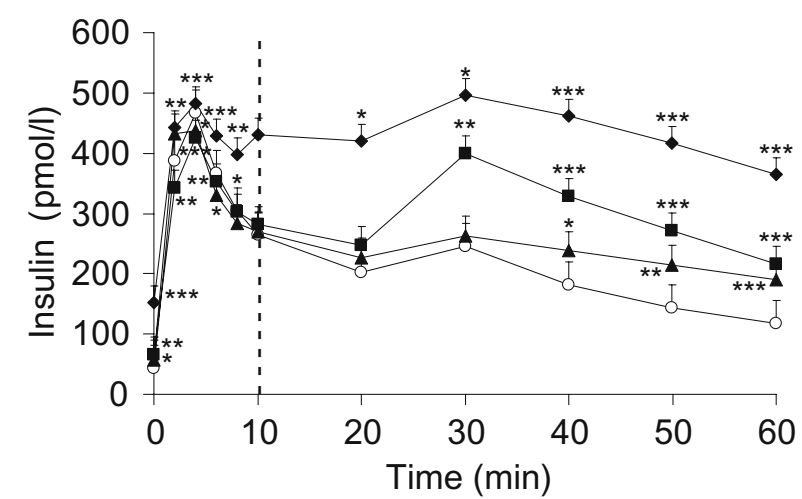

Fig. 2 Glucose (a) and insulin (b) levels in participants with different glucose tolerance during IVGTT. First and second phase are separated by a dotted line. Circles, normal glucose tolerance; squares, isolated IFG; triangles, isolated IGT; diamonds, combined IFG and IGT. $p$ values are adjusted for age, sex, BMI, centre and familial relationship. ${ }^{*} p<0.05, * * p<0.01, * * * p<0.001$ vs NGT 
a

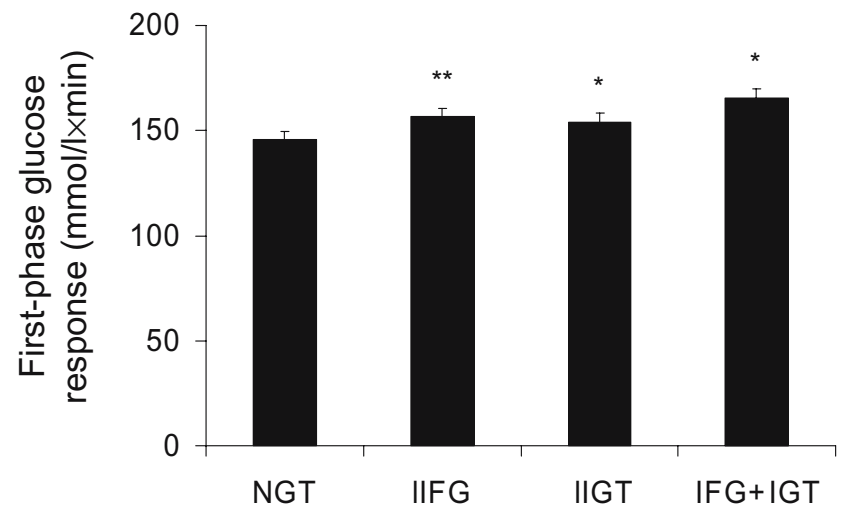

C

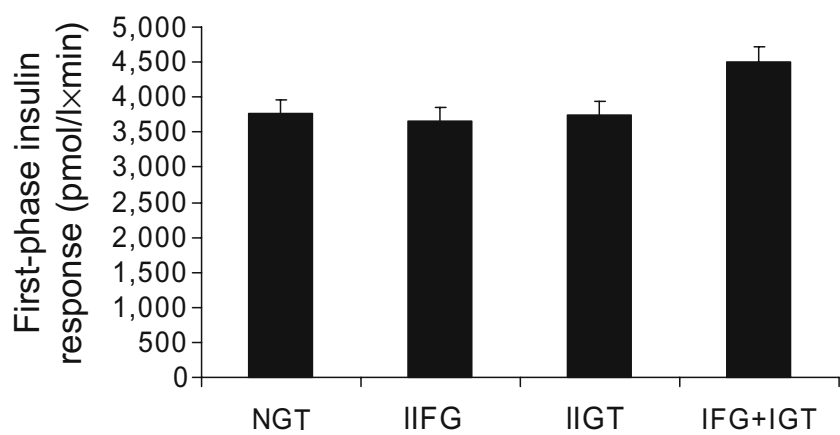

e

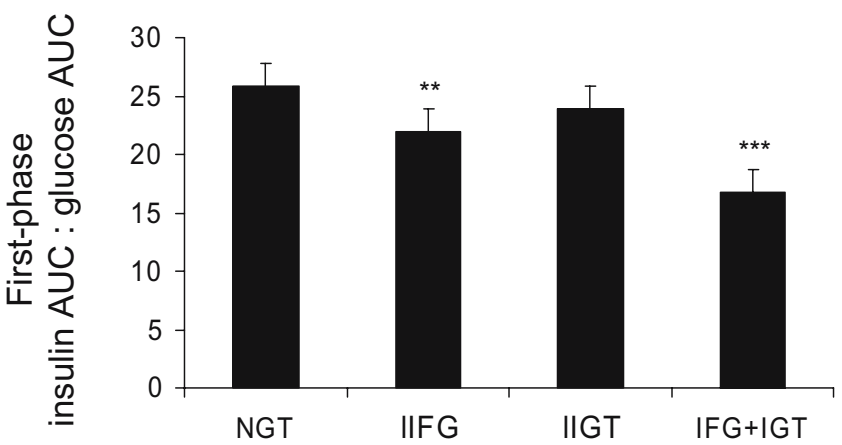

Fig. 3 First-phase (a) and second-phase (b) glucose levels under the curve, followed by first- (c) and second-phase (d) insulin levels under the curve during the IVGTT, and ratio of insulin and glucose areas under the curve during the first (e) and second (f) phase of the IVGTT. $p$ values for glucose measurements are adjusted for age, sex, BMI,

tion (IVGTT) and insulin action (hyperinsulinaemic clamp) and was carried out in Pima Indians [10]. The results showed that individuals with isolated IFG and isolated IGT had a comparable impairment in insulin action, whereas those with isolated IFG had greater impairment in firstphase insulin release, combined with increased endogenous glucose output as compared with individuals with NGT. Participants with both IFG/IGT had even more profound abnormalities in insulin action, insulin secretion and endogenous glucose output [10]. Although that study by b

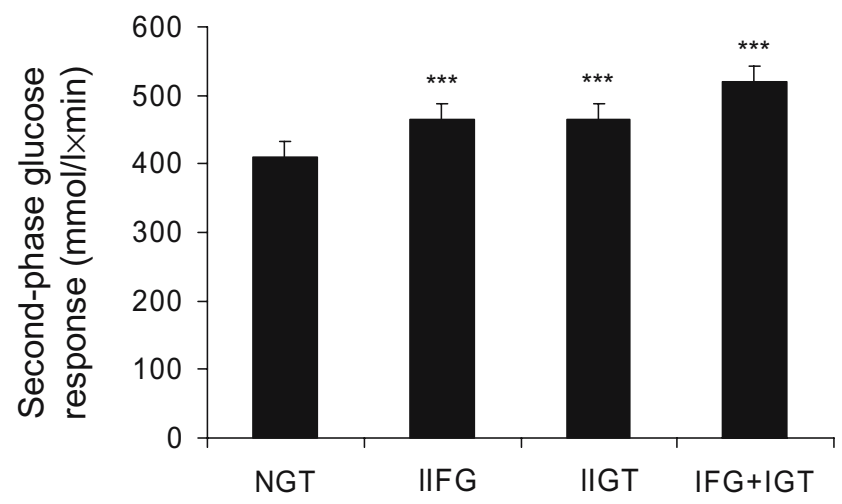

d

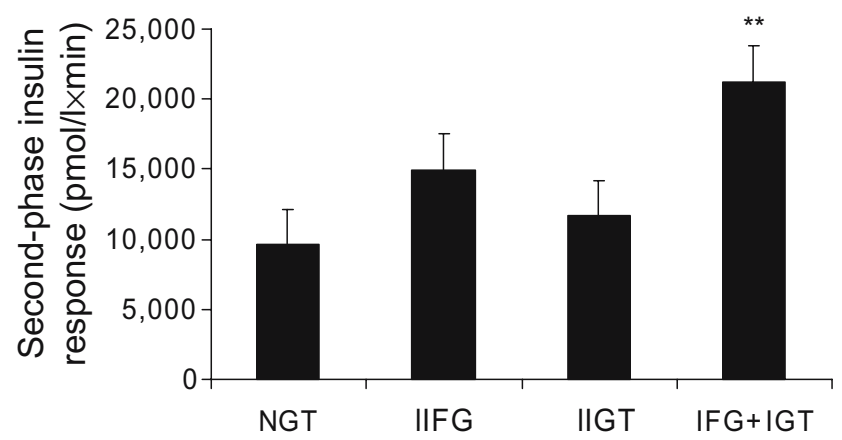

f

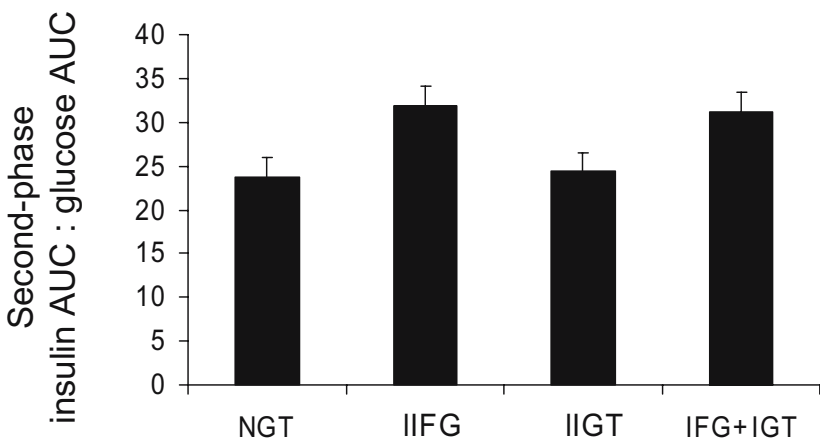

centre and familial relationship. Those for insulin measurements are adjusted for age, sex, BMI, insulin sensitivity (measured by clamp), centre and familial relationship. ${ }^{*} p<0.05,{ }^{* *} p<0.01,{ }^{* * *} p<0.001$ vs NGT. IIFG, isolated IFG; IIGT, isolated IGT

Weyer et al. [10] was quite small and, for instance, only included 11 participants in the isolated IFG group, these results are in general agreement with our findings in a large group of white first-degree relatives.

Three other studies using accurate measurements of insulin secretion and insulin resistance have been published. Van Haeften et al. [14] assessed beta cell function and insulin sensitivity with hyperglycaemic clamps in 24 participants with IFG, 15 type 2 diabetic participants and 280 participants with NGT. First-phase $(0-10 \mathrm{~min})$ and 
a

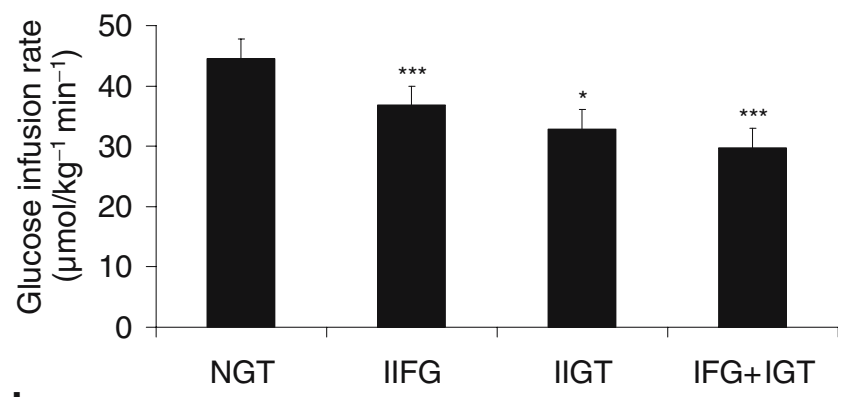

b

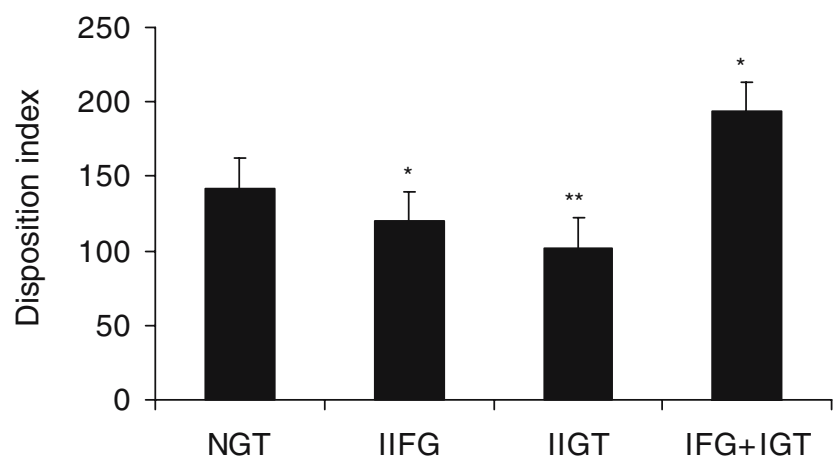

Fig. 4 Insulin sensitivity measured by clamp (a) and disposition index (b). $p$ values are adjusted for age, sex, BMI, centre and familial relationship. ${ }^{*} p<0.05, * * p<0.01, * * * p<0.001$, all vs NGT. IIFG, isolated IFG; IIGT, isolated IGT

second phase (140-180 $\mathrm{min})$ insulin release was lower in participants with IFG than in participants with NGT. Participants with IFG and IGT had lower insulin sensitivity than those with isolated IGT. Recently, the same group of investigators [19] used the hyperglycaemic clamp to study 21 individuals with isolated IFG, 61 individuals with isolated IGT and 240 individuals with NGT. They showed that participants with isolated IFG had similar fasting insulin concentrations to those with NGT despite their hyperglycaemia. Both first- and second-phase insulin responses were reduced by $30 \%$. Participants with isolated IGT had about $50 \%$ greater second-phase insulin response than participants with isolated IFG. The authors concluded that IFG is due to impaired basal insulin secretion and preferential resistance to the suppressive effect of insulin on glucose production, whereas IGT mainly results from reduced second-phase insulin release and peripheral insulin resistance.

Festa et al. [16] used a frequently sampled IVGTT to evaluate insulin sensitivity and insulin secretion. In a large cohort of individuals, they showed that participants with isolated IGT are more insulin-resistant than individuals with isolated IFG, whereas participants with isolated IFG had impaired insulin secretion. Another study [20] investigated the metabolic profile of 32 participants with IFG compared with 28 participants with NGT. In that study, insulin secretion and insulin sensitivity were normal in participants with isolated IFG, whereas insulin secretion and insulin action were impaired in participants with IGT. Taken together, it is clear that defects both in insulin secretion and insulin action contribute to postprandial hyperglycaemia. Furthermore, postprandial glucose metabolism is essentially normal in participants with isolated IFG, which might indicate a lower risk of progression to overt diabetes.

As discussed above, even studies [10, 14, 16, 19, 20] where a detailed metabolic characterisation of the participants was performed have led to somewhat inconsistent results. However, even more contradictory results have been published from studies based on indirect methods, i.e. only fasting glucose and insulin levels and results from an OGTT, to evaluate insulin sensitivity and insulin secretion. For example, Hanefeld et al. [15] compared measures of insulin sensitivity (evaluated by HOMA-IR) and insulin secretion (evaluated by the insulinogenic index) in partici-

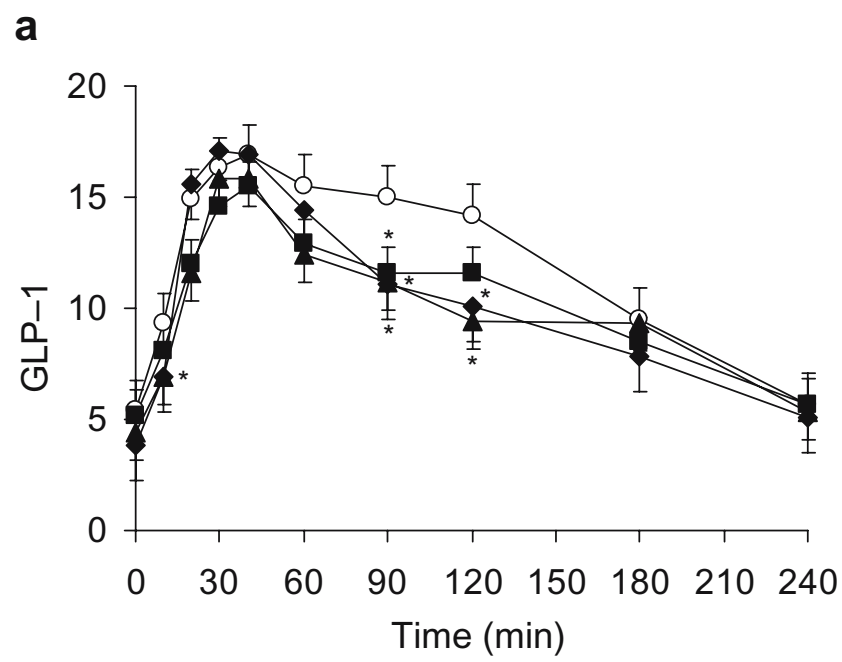

b

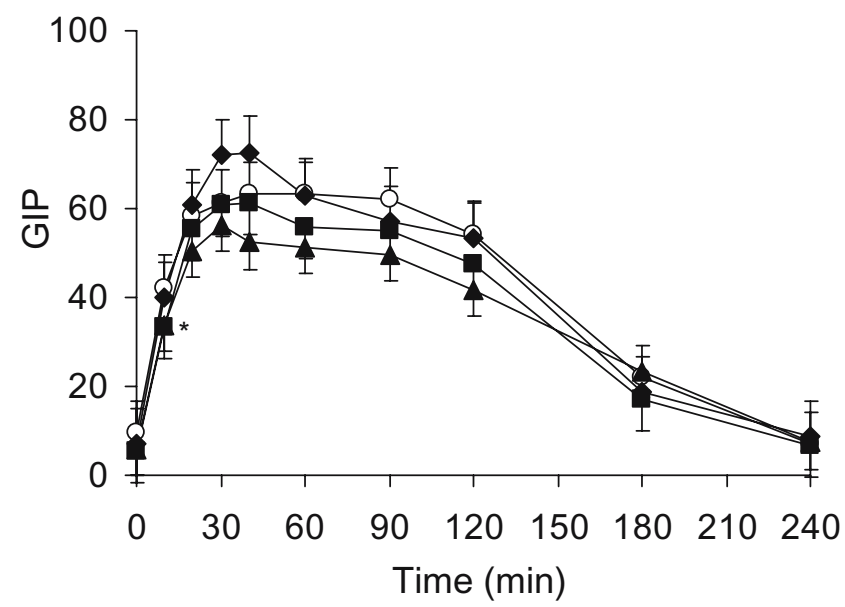

Fig. 5 GLP-1 (a) and GIP (b) levels, measured in 278 participants during a prolonged OGTT. Circles, normal glucose tolerance; squares, isolated IFG; triangles, isolated IGT; diamonds, combined IFG and IGT. $p$ values are adjusted for age, sex, BMI and familial relationship. $* p<0.05$ vs NGT 
pants with NGT $(n=367)$, IFG $(n=90)$, IGT $(n=101)$, and both IFG and IGT $(n=106)$. They found that participants with isolated IFG and IFG/IGT were more insulin-resistant than those with IGT. Insulinogenic index indicated that all participants with abnormal glucose tolerance had impaired insulin secretion compared with those who had NGT. Another study [11] included 3,086 participants with NGT, 537 with IFG, 493 with IGT and 303 with both IFG and IGT, recruited from families that had type 2 diabetic members in the Botnia Study. The authors concluded that IFG is characterised by insulin resistance and other features of the metabolic syndrome, whereas participants with IGT have impaired insulin secretion.

In the present study, we demonstrated in a large cohort of offspring of type 2 diabetic patients that participants with isolated IFG are characterised by: (1) impaired basal insulin secretion when related to degree of insulin sensitivity; (2) reduced glucose-adjusted first-phase insulin response; (3) normal second-phase insulin release; and (4) reduced insulin sensitivity compared with NGT individuals. By contrast, participants with isolated IGT had unchanged basal insulin secretion and first- and second-phase insulin release, combined with increased insulin resistance. Compared with previously published results obtained using advanced techniques to estimate insulin secretion and insulin action [10, $16,19]$, our findings are similar to two of them [10, 16], showing that isolated IFG is characterised by impaired firstphase insulin release and increased insulin resistance. Basal insulin secretion was evaluated in only one study [19], which, similarly to ours, demonstrated that basal insulin secretion was decreased in participants with isolated IFG.

Although previous studies have shown inconsistencies, some general conclusions can be drawn about IFG vs IGT. All groups of glucose intolerance are characterised by insulin resistance which may be due to genetic factors and/ or glucose toxicity. However, individuals with IGT have a more pronounced degree of insulin resistance. In contrast, IFG is characterised by a more pronounced beta cell defect when related to the ambient glucose levels and degree of insulin sensitivity. To what extent this can be due to a changed set-point for glucose, as previously suggested [20], or to an inherent abnormality in the beta cells is currently unclear. It is also clear that there must be a reduced hepatic sensitivity to insulin in IFG, as also previously shown [10, 20]. Since it was recently found that glucagon levels are raised in individuals with IFG [20], an interesting possibility is that IFG is characterised by an insensitivity to GLP-1, both in terms of insulin secretion and ability to suppress glucagon release. Interestingly, a reduced ability to respond to GLP-1 has recently been shown in individuals carrying the risk gene TCF7L2 [31], which is also associated with impaired insulin release. Thus, clinical GLP-1 resistance may well be an important prediabetic state.
Our study has limitations. All our participants were offspring of type 2 diabetic probands and, therefore, we cannot be certain that our results can be generalised to participants without a family history of type 2 diabetes. However, considering the strong genetic influence on risk for type 2 diabetes, we feel that this design is more a strength than a weakness. Second, our data included five different white populations, and so genetic differences between the populations might have influenced the results. However, previous work has not shown an important role of ethnicity for the phenotypic differences between IFG and IGT [16]. Finally, hepatic glucose production was not measured. We cannot, therefore, draw any conclusions about the role of the liver in the pathophysiology of IFG and IGT, which has, however, been previously examined in similar groups $[10,20]$.

In summary, our results suggest that the primary mechanism leading to hyperglycaemia in participants with isolated IFG is impaired basal and first-phase insulin secretion, whereas in isolated IGT, insulin resistance is the primary mechanism leading to postglucose load hyperglycaemia. Ongoing follow-up studies of the EUGENE2 cohorts will provide information about the future risk of developing type 2 diabetes in relation to phenotype and genotype.

Acknowledgements This work was supported by the European Community's FP6 EUGENE2 (LSHM-CT-2004-512013) and other national funds to the investigators. The EUGENE2 consortium, which is funded by the European Commission, consists of the following laboratories: U. Smith (Sweden), M. Laakso (Finland), H. Häring (Germany), G. Sesti (Italy), O. Pedersen (Denmark), J. Zierath (Sweden), H.-G. Joost (Germany), F. Beguinot (Italy), E. Van Obberghen (France), J. Auwerx (France), F. Bosch (Spain) and P. Lind (Sweden) (see www.eugene2.com).

Duality of interest The authors declare that there is no duality of interest associated with this manuscript.

\section{References}

1. Lillioja S, Mott DM, Spraul M et al (1993) Insulin resistance and insulin secretory dysfunction as precursors of non-insulin-dependent diabetes mellitus. Prospective studies of Pima Indians. N Engl J Med 329:1988-1992

2. Haffner SM, Miettinen H, Gaskill SP, Stern MP (1995) Decreased insulin secretion and increased insulin resistance are independently related to the 7-year risk of NIDDM in Mexican-Americans. Diabetes 44:1386-1391

3. Curry DL, Bennett LL, Grodsky GM (1968) Dynamics of insulin secretion by the perfused rat pancreas. Endocrinology 83:572-584

4. Gerich JE (2002) Is reduced first-phase insulin release the earliest detectable abnormality in individuals destined to develop type 2 diabetes? Diabetes 51(Suppl 1):S117-S121

5. Bergman RN, Phillips LS, Cobelli C (1981) Physiologic evaluation of factors controlling glucose tolerance in man: measurement of insulin sensitivity and beta cell glucose sensitivity from the response to intravenous glucose. J Clin Invest 68:1456-1467 
6. Kahn SE, Prigeon RL, McCulloch DK et al (1993) Quantification of the relationship between insulin sensitivity and beta cell function in human subjects. Evidence for a hyperbolic function. Diabetes 42:1663-1672

7. No authors listed (1997) Report of the Expert Committee on the diagnosis and classification of diabetes mellitus. Diabetes Care 20:1183-1197

8. Genuth S, Alberti KG, Bennett P et al (2003) Follow-up report on the diagnosis of diabetes mellitus. Diabetes Care 26:3160-3167

9. Alberti KG, Zimmet PZ (1998) Definition, diagnosis and classification of diabetes mellitus and its complications. Part 1: Diagnosis and classification of diabetes mellitus provisional report of a WHO consultation. Diabet Med 15:539-553

10. Weyer C, Bogardus C, Pratley RE (1999) Metabolic characteristics of individuals with impaired fasting glucose and/or impaired glucose tolerance. Diabetes 48:2197-2203

11. Tripathy D, Carlsson M, Almgren P et al (2000) Insulin secretion and insulin sensitivity in relation to glucose tolerance: lessons from the Botnia Study. Diabetes 49:975-980

12. Davies MJ, Raymond NT, Day JL, Hales CN, Burden AC (2000) Impaired glucose tolerance and fasting hyperglycaemia have different characteristics. Diabet Med 17:433-440

13. Kim DJ, Lee MS, Kim KW, Lee MK (2001) Insulin secretory dysfunction and insulin resistance in the pathogenesis of Korean type 2 diabetes mellitus. Metabolism 50:590-593

14. van Haeften TW, Pimenta W, Mitrakou A et al (2002) Disturbances in beta cell function in impaired fasting glycemia. Diabetes 51(Suppl 1):S265-S270

15. Hanefeld M, Koehler C, Fuecker K, Henkel E, Schaper F, Temelkova-Kurktschiev T (2003) Insulin secretion and insulin sensitivity pattern is different in isolated impaired glucose tolerance and impaired fasting glucose: the risk factor in Impaired Glucose Tolerance for Atherosclerosis and Diabetes study. Diabetes Care 26:868-874

16. Festa A, D’Agostino R Jr, Hanley AJ, Karter AJ, Saad MF, Haffner SM (2004) Differences in insulin resistance in nondiabetic subjects with isolated impaired glucose tolerance or isolated impaired fasting glucose. Diabetes 53:1549-1555

17. Wasada T, Kuroki H, Katsumori K, Arii H, Sato A, Aoki K (2004) Who are more insulin resistant, people with IFG or people with IGT? Diabetologia 47:758-759

18. Nishi Y, Fukushima M, Suzuki H et al (2005) Insulin secretion and insulin sensitivity in Japanese subjects with impaired fasting glucose and isolated fasting hyperglycemia. Diabetes Res Clin Pract 70:46-52
19. Meyer C, Pimenta W, Woerle HJ et al (2006) Different mechanisms for impaired fasting glucose and impaired postprandial glucose tolerance in humans. Diabetes Care 29:1909-1914

20. Bock G, Dalla Man C, Campioni M et al (2006) Pathogenesis of pre-diabetes: mechanisms of fasting and postprandial hyperglycemia in people with impaired fasting glucose and/or impaired glucose tolerance. Diabetes 55:3536-3549

21. DeFronzo RA, Tobin JD, Andres R (1979) Glucose clamp technique: a method for quantifying insulin secretion and resistance. Am J Physiol 237:E214-E223

22. Tripathy D, Wessman Y, Gullstrom M, Tuomi T, Groop L (2003) Importance of obtaining independent measures of insulin secretion and insulin sensitivity during the same test: results with the Botnia clamp. Diabetes Care 26:1395-1401

23. Krarup T, Madsbad S, Moody AJ et al (1983) Diminished immunoreactive gastric inhibitory polypeptide response to a meal in newly diagnosed type I (insulin-dependent) diabetics. J Clin Endocrinol Metab 56:1306-1312

24. Orskov C, Rabenhoj L, Wettergren A, Kofod H, Holst JJ (1994) Tissue and plasma concentrations of amidated and glycineextended glucagon-like peptide I in humans. Diabetes 43:535-539

25. Deacon CF, Pridal L, Klarskov L, Olesen M, Holst JJ (1996) Glucagon-like peptide 1 undergoes differential tissue-specific metabolism in the anesthetized pig. Am J Physiol 271:E458-E464

26. Matthews DR, Hosker JP, Rudenski AS, Naylor BA, Treacher DF, Turner RC (1985) Homeostasis model assessment: insulin resistance and beta cell function from fasting plasma glucose and insulin concentrations in man. Diabetologia 28:412-419

27. Laakso M (2005) Prevention of type 2 diabetes. Curr Mol Med $5: 365-374$

28. No authors listed (1999) Glucose tolerance and mortality: comparison of WHO and American Diabetes Association diagnostic criteria. The DECODE study group. European Diabetes Epidemiology Group. Diabetes Epidemiology: Collaborative analysis Of Diagnostic criteria in Europe. Lancet 354:617-621

29. Nauck M, Stockmann F, Ebert R, Creutzfeldt W (1986) Reduced incretin effect in type 2 (non-insulin-dependent) diabetes. Diabetologia 29:46-52

30. Toft-Nielsen MB, Damholt MB, Madsbad S et al (2001) Determinants of the impaired secretion of glucagon-like peptide1 in type 2 diabetic patients. J Clin Endocrinol Metab 86:37173723

31. Lyssenko V, Lupi R, Marchetti P et al (2007) Mechanisms by which common variants in the TCF7L2 gene increase risk of type 2 diabetes. J Clin Invest 117:2155-2163 